\title{
Dissecting the Role of Synaptic Proteins with CRISPR
}

\author{
Salvatore Incontro, Cedric S. Asensio, and Roger A. Nicoll
}

\begin{abstract}
A significant step forward in the study of synaptic physiology is the application of single cell genetic modifications. In this landscape, the dissection of the role of single proteins or, more significantly, their subunits and sub-domains has increased enormously the basic knowledge of synaptic function. CRISPR/Cas9 is a recently developed genome-editing tool that can be used to inactivate or modify genes of interest. Its ease of implementation and affordable cost, combined with its high efficiency, make it a very valuable tool to study various biological processes. The application of this technique in addition to previous genetic approaches vastly simplifies and accelerates the study of specific synaptic proteins. Here we illustrate different ways that CRISPR/Cas9 can be used in the study of synaptic properties.
\end{abstract}

\section{Introduction}

Over the last two decades, the combination of pharmacology and genetics has been instrumental in our current understanding of the molecular mechanisms controlling diverse neuronal processes. The development of gene targeting through homologous recombination enabled the generation of knockout (KO) transgenic animals, and this ability to completely inactivate genes of interest for synaptic transmission has provided invaluable information about their function. Although germline gene deletion has dramatically advanced our knowledge, the approach suffers from two main limitations: the deletion can be embryonically lethal if the gene is essential or it can lead to physiological compensation during development, masking the real importance of the studied protein. In addition, the development of transgenic animals represents a significant investment of both cost and time.

The more recent development of RNAi has provided an easier and faster way to inactivate proteins, but use of this technique is limited by the efficiency of

\footnotetext{
S. Incontro $(\varangle) \cdot$ R.A. Nicoll

Department of Cellular and Molecular Pharmacology, University of California, San Francisco,

San Francisco, CA 94158, USA

e-mail: Salvatore.Incontro@ucsf.edu

C.S. Asensio

Department of Biological Sciences, University of Denver, Denver, CO 80120, USA
} 
knockdown. Indeed, in the case of incomplete knockdown, residual protein can lead to serious misinterpretation. In addition, off-target effects present an important concern. Indeed, it has been observed that RNAi manipulation can affect the morphology of single spines (Alvarez et al. 2006), suggesting some general non-specific effects of RNAi in neurons.

More recently, the development of conditional knockout (cKO) technology has offered an interesting alternative to the limitations associated with both germline $\mathrm{KO}$ and RNAi approaches. Indeed, the $\mathrm{cKO}$ approach relies on the generation of transgenic mice with LoxP sites flanking a gene of interest. The subsequent sparse transfection of Cre recombinase in brain slices derived from these LoxP animals results in the removal of the gene of interest from a few neurons and offers a more controllable way to compare genetically manipulated neurons to controls by dual cell patch clamp (Adesnik et al. 2008; Pluck 1996; Hayashi et al. 2000; Schnell et al. 2002; Sauer and Henderson 1988; Tsien et al. 1996). This method is particularly powerful for studying proteins that are essential for the maintenance of synaptic equilibrium. For example, this genetic inactivation approach has been used successfully to determine the function of single subunits of the excitatory post-synaptic AMPA and NMDA receptors (Lu et al. 2009; Gray et al. 2011) and the role of the different isoforms of the SNARE protein complex machinery at the pre-synapse (Hun et al. 2014; Han et al. 2011; Maximov et al. 2007). Nevertheless, the same time and cost considerations associated with the development of germline KO animals apply to the Cre-LoxP system.

\section{Genome Editing Using CRISPR/Cas9}

Genome editing generally relies on the guided activity of endonucleases to generate double-strand breaks at a specific location in the genomic DNA in order to modify it. In eukaryotic cells, there are two main types of DNA repair mechanism following double-strand DNA breaks: non-homologous end joining (Barnes 2001; Lieber 2010) and homologous recombinational repair. Non-homologous end joining is generally accompanied by the loss/gain of nucleotides such as deletions, insertions or nucleotide substitutions in the repaired region, thus often leading to inactivation of the targeted gene. On the other hand, homologous recombination uses the complementary DNA as a template to repair the double-strand DNA breaks. The outcome of this type of repair is generally more precise and controllable, so it can be used to either introduce point mutations or knock-in entire proteins through the use of a repair template.

CRISPR/Cas9 is a recently developed genome-editing technique arising from a bacterial adaptive defense system against invading plasmids or phages. The term CRISPR stands for Clusters of Regularly Interspaced Short Palindromic Repeats. These CRISPR loci are found in bacteria and are composed of partially palindromic non-coding repeats that are separated by non-repetitive spacers of similar length. These repeats and spacers are transcribed into one long RNA transcript that is further processed into smaller CRISPR RNAs by endonucleases encoded by 
CRISPR-associated (Cas) genes flanking the CRISPR loci (Ishino et al. 1987; Nakata et al. 1989; Pourcel et al. 2005; Jansen et al. 2002). Each individual CRISPR RNA corresponds to one repetitive unit of the original CRISPR array and will guide Cas nucleases to their target by recognizing the homologous DNA region. To work as a defense mechanism, new spacers deriving from invading plasmids or phages are added to the CRISPR locus (Bolotin et al. 2005; Pourcel et al. 2005). Once transcribed and processed into CRISPR RNAs, these new spacers then serve as memory signatures of past invasions, enabling the bacteria to recognize and cleave foreign DNAs (Makarova et al. 2006).

As a genome-editing tool, the technique relies on the nuclease activity of one of these Cas genes (SpCas9) derived from Streptococcus pyogenes. The activity of SpCas9 depends on two of these processed RNAs: a CRISPR RNA and a transactivating CRISPR RNA, which combine to form an RNA complex. The critical features of this complex are the presence of a double-stranded RNA structure at the $3^{\prime}$ end that physically interacts with SpCas9 and a 20-nucleotide sequence at the $5^{\prime}$ end, which guides the binding of SpCas9 to the target DNA by homology (Jinek et al. 2012). In addition, the proper targeting of SpCas9 requires the presence of a short sequence of the complementary sequence on the target DNA. This sequence is called the protospacer adjacent motif (PAM) and, in the case of SpCas9, consists of a nucleotide triplet (NGG). Importantly, in the absence of the PAM, Cas9 cannot recognize target sequences even when they are fully complementary to the guide RNA (Sternberg et al. 2014). By engineering chimeric single RNAs consisting of a fusion between the trans-activating CRISPR RNAs and the CRISPR RNAs, it becomes possible to mimic the natural RNA complex and to control the targeting of Cas9 to a specific region of the genome by simply changing the $5^{\prime}$ complementary sequence of the RNA complex (Jinek et al. 2012; Jiang et al. 2013). This so-called guide RNA consists of 20 nucleotides complementary to the region of interest, whose only requirement for its design is the presence of a PAM at the $3^{\prime}$ end (on the target DNA). As this motif is very frequent in eukaryotic genomes (Wu et al. 2014), it becomes possible to target virtually any gene of interest, making CRISPR/Cas9 a very powerful and promising tool for basic research as well as for potential therapeutic use. Unlike other genome-editing tools requiring the design and generation of specific nucleases for each target site, CRISPR/Cas9 relies on a simple two-component system: Cas9 and a target-specific guide RNA.

\section{Practical Considerations for the Use of CRISPR/Cas9}

The careful design of guide RNAs represents one of the key steps in successful use of CRISPR/Cas9. The first step consists of choosing the best region to target within the gene of interest and subsequently scanning this sequence for the presence of PAM motifs. When selecting guide RNAs, it is important to consider the possibility that the non-homologous end joining repair mechanism might lead to in-frame deletions resulting from Cas9 cleavage in position -3 from the PAM (see Figs. 1 and 2). If 

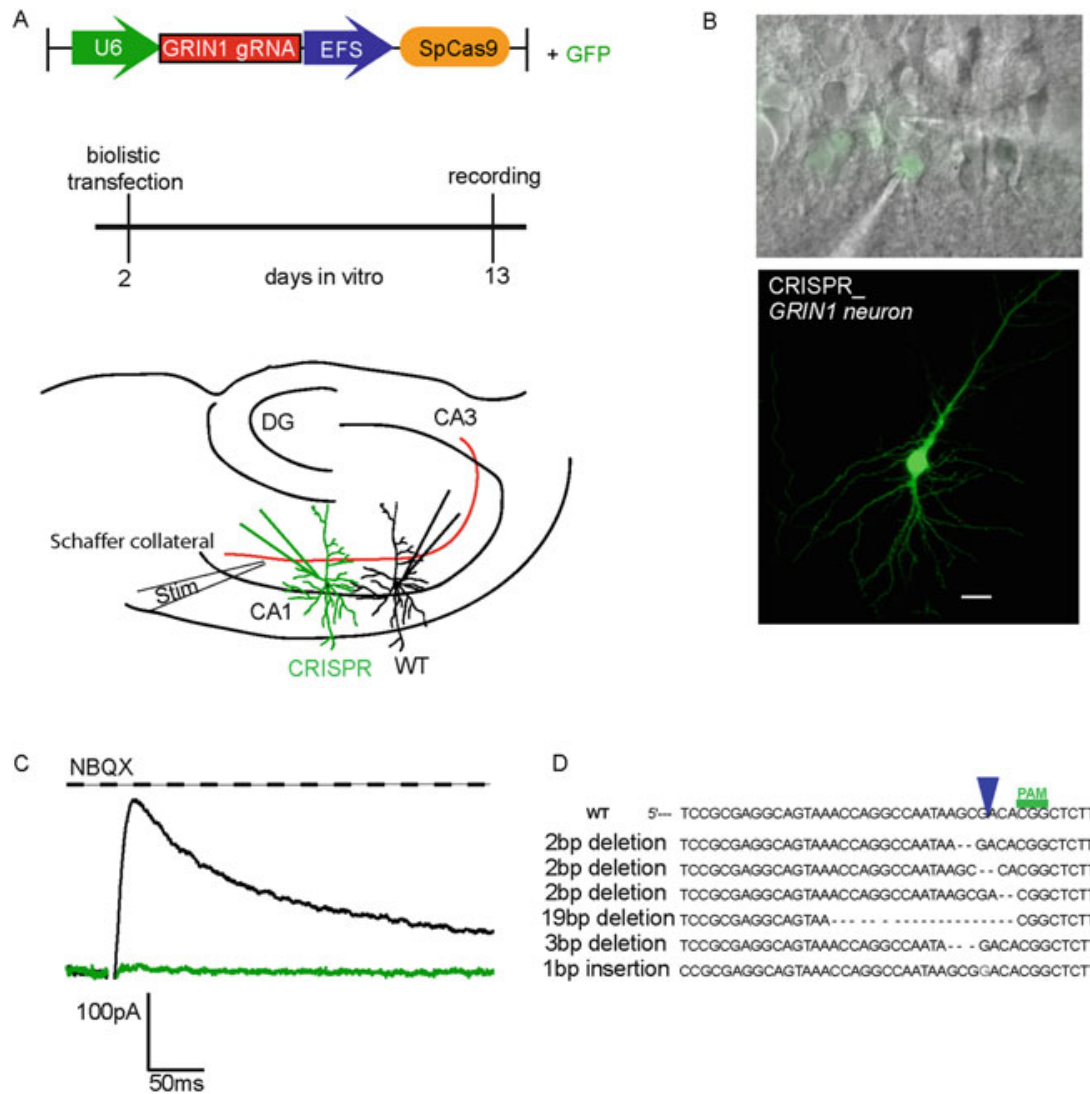

D

WT 5-- TCCGCGAGGCAGTAAACCAGGCCAATAAGCCACACGGGCTCTTGGA - 3. 2bp deletion TOCGCGAGGCAGTAAACCAGGCCAATAA.. GACACGGCTCTTGGA 5 2bp deletion TOCGOGAGGCAGTAAACCAGGCCAATAAGC ... CACGGCTCTTGGA 1 $2 \mathrm{bp}$ deletion TOCGOGAGGCAGTAAACCAGGCCAATAAGCGA -. CGGCTCTTGGA 1 19bp deletion TOCGOGAGGCAGTAA.................... СGGCTCTTGGA 1 3bp deletion TOCGCGAGGCAGTAAACCAGGCCAATA -... GACACGGCTCTTGGA 1 1bp insertion $\triangle$ COCGAGGCAGTAAACCAGGCCAATAAGCG CACACGGCTCTTGGA 2

Fig. 1 (a) Timeline of the CRISPR_GRIN1 GluN1 deletion experimentation and scheme of dual whole-cell voltage-clamp recording in organotypic hippocampal slices of a biolistically transfected pX330 CRISPR_GRIN1 neuron and a neighboring wild-type neuron. (b) Representative phase contrast + epifluorescence image of the CA1 region of a hippocampal slice and confocal image of a CRISPR_GRIN1 neuron co-transfected with a FUGW-EGFP plasmid. Scale bar: $20 \mu \mathrm{M}$. (c) Sample traces of NMDAR-evoked EPSCs, from a transfected CRISPR_GRIN1 neuron and a neighboring control in the presence of NBQX $(10 \mu \mathrm{M})$. (d) Targeted GRINl region and types of insertions or deletions in the DNA after infecting dissociated hippocampal neurons with lentiCRISPR GRIN1 (adapted from Fig. 1 of Incontro et al. 2014)

structural information about the protein is available, it can be used to select a region that is essential for its stability. Unfortunately, for most proteins this information does not exist, and the best strategy to efficiently inactivate the gene of interest is usually to target one of the first exons in order to minimize the chance of generating a truncated, functional protein. When potential $20 \mathrm{bp}$ sequences have been selected, several on-line tools enable users to find sequences with the lowest probabilities for off-target effects based on their lack of similarities to other parts of the genome.

The rescue experiments also provide a powerful tool to assess the role of specific protein domains. By transfecting cDNAs with point mutations or domain deletions, it 


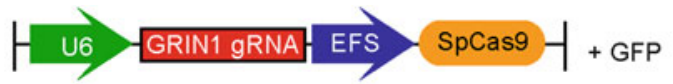

A

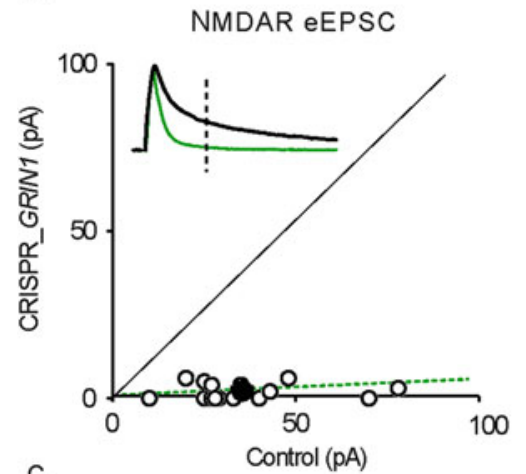

C

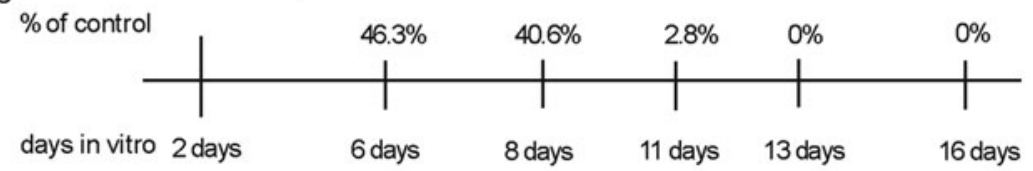

B

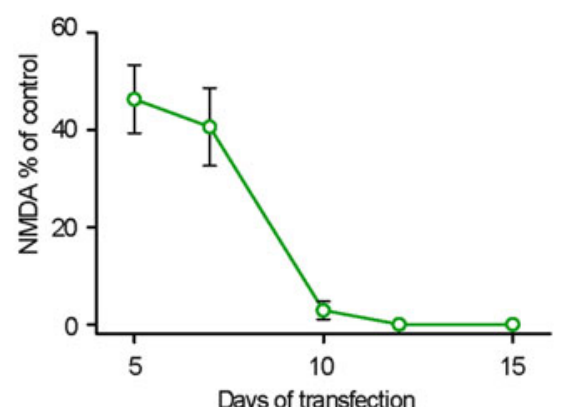

Days of transfection
6 days 8 days 11 days 13 days 16 days

U6 GRIN1 gRNA EFS SpCas9- + GluN1 cDNA

D

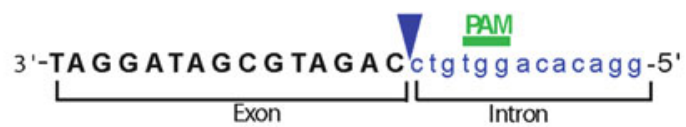

E

$\mathrm{F}$

NMDAR EEPSC

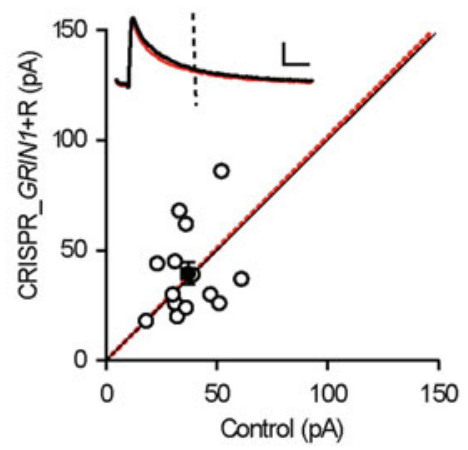

AMPAR EEPSC

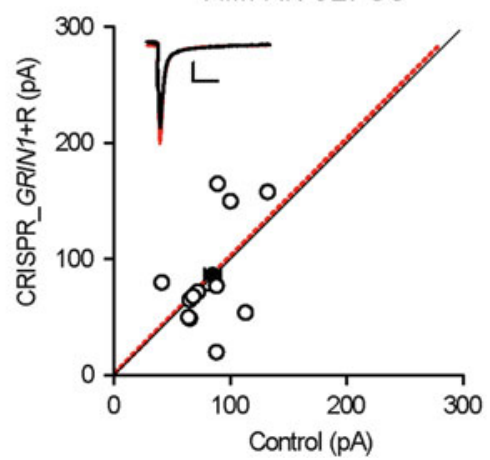

Fig. 2 (a) Scatterplot and sample traces of NMDAR eEPSCs in 14 days transfected CRISPR/Cas9 and neighboring control neurons. Open circles represent amplitudes of NMDA EPSCs for single cells; filled circle represent the mean. (b) Time course of NMDAR eEPSC 5, 7, 10, and 15 days after transfection. The evoked currents are eliminated after 12 days. (c) Scheme of the time course 
becomes possible to assess their significance for the biological process being studied, similar to what has been done with conditional KO animals (Herring et al. 2013).

\section{The Use of CRISPR/Cas9 in Neurons: Proof of Concept}

To test the potential of the CRISPR/Cas9 technology in neuroscience, we have performed a proof of concept study aimed at assessing its efficiency to inactivate synaptic proteins. In particular, we focused on two fundamental subunits of the ionotropic glutamate receptors in hippocampal slice cultures: the GluN1 subunit of NMDA receptors and the GluA2 subunit of AMPA receptors. We began by designing two different guide RNAs targeting the extracellular part of the GluN1 subunit, and we selected guide RNAs with a score $>70 \%$ corresponding to a low probability of off-target effects according to the MIT online CRISPR design tool. We then co-introduced by biolistic transfection into hippocampal slices a plasmid encoding both Cas9 and one of the guide RNAs together with a plasmid encoding GFP as described previously for cKOs (Adesnik et al. 2008). As the efficiency of this transfection approach is modest, the system as a whole is only minimally perturbed and it becomes possible to directly compare recordings obtained simultaneously from a target, transfected neuron (GFP positive) and a control, untransfected neighbor neuron (GFP negative; Fig. 1a, b). NMDA currents (eEPSCs) were completely abolished in $100 \%$ of the pyramidal neurons analyzed (Fig. 1c). Consistent with previous results (Adesnik et al. 2008), we also observed a compensatory increase in AMPA currents. We sequenced the DNA region targeted by Cas9 after PCR amplification of the genomic DNA and found the presence of various small insertions and deletions (indels) creating frameshifts in $90 \%$ of the cases (Fig. 1d). This first set of experiments thus suggests that Cas9 is able to efficiently inactivate genes in adult pyramidal neurons by creating double-strand DNA breaks, which are repaired by the non-homologous end joining system. The extreme efficiency that we observed contrasts with the efficiency reported by others using different cell types and is somewhat surprising, but probably reflects the postmitotic nature of adult pyramidal neurons. In contrast to dividing cells, which rapidly dilute the Cas9 machinery, neurons have the ability to maintain high levels of the CRISPR/Cas9 components for a longer period. Under these conditions, Cas9

Fig. 2 (continued) and percentages of control of NMDAR-evoked EPSCs after CRISPR_GRIN1 biolistic transfection. (d) Scheme of the targeted region in the GRIN1 gene. The guide RNA not including the PAM region is shown in bold; the intronic part of the gene, which includes the PAM region, is shown in blue. (e) Scatterplot and sample traces of NMDA eEPSCs from a transfected CRISPR_GRIN1 neuron + GluN1 cDNA and a neighboring control neuron. Scale bar: 50 pA and $50 \mathrm{~ms}$. (f) Scatterplot and sample traces of AMPA eEPSCs from a transfected CRISPR_GRIN1 neuron + the GluN1 cDNA and a neighboring control neuron. Scale bar: $50 \mathrm{pA}$ and $50 \mathrm{~ms}$ (Adapted from Figs. 2 and 3 of Incontro et al. 2014) 
will presumably have sufficient time to cut the targeted region until it can no longer be properly repaired.

To rule out the existence of off-target effects, we also performed rescue experiments by transfecting a GluN1 cDNA. Re-introduction of the deleted subunit by co-transfection fully rescued the phenotype (Fig. 2).

In a subsequent part of our project, our goal was to target multiplex genes. As a proof of concept we repeated the same experiment with the single GluN1 and GluA2 subunits, this time co-transfecting the two plasmids containing target gRNAs. We observed a complete deletion of both subunits with a complete rectification for AMPA receptors (due to the loss of GluA2) and no NMDA eEPSCs (Fig. 3).

Another issue regards the possibility of studying an effect of a protein deletion in vivo. The advent of Cas9 opens a very exciting new concept-we can now inject gRNAs to target potentially any protein in a wild-type (WT) background (co-transfecting with Cas9 plasmids) or in Cas9 knock-in animals (Platt et al. 2014). For example the use of the AMPA receptors triple floxed mouse has been very important for understanding every single subunit's contribution to the structure and function of glutamatergic excitatory synapses. Now we can reproduce these results in a few weeks (compared to years to create Cre-Flox lines and to cross them), optimizing time and cost enormously (Fig. 4).

The possibility of expressing the protein of interest in a $\mathrm{KO}$ background enables one to study the function of specific domains in the synaptic context. This approach can be instrumental to the understanding of synaptic proteins that are involved in neurological diseases.

\section{Conclusions and Future Perspectives}

The field of biological engineering has seen the rapid development of several novel technologies over the last few years, and neuroscience has embraced many of them to explore the function of synaptic proteins in a more precise and definitive way. Recent development of the CRISPR/Cas9 technology provides a simpler and faster alternative for studying synaptic proteins by removing the time and cost associated with the generation of genetically manipulated animals. Indeed, the approach can be used for the inactivation of target genes, but it also enables one to determine the significance of particular protein domains by performing rescue experiments, as discussed above. In addition, it is possible to place the expression of Cas9 under the control of a neuronal specific promoter for use in vivo, similar to what has been done with Cre previously (Gray et al. 2011; Lu et al. 2009; Schnell et al. 2002). Finally, another powerful feature of the CRISPR/Cas9 technology is the ability to easily inactivate several proteins at once using multiplex guide RNAs.

How can one determine that the cleavage has indeed happened? The importance of this validation is best illustrated in a recent short report (Straub et al. 2014) in which the authors performed in utero electroporation in mice to inactivate GluN1. Recording from hippocampal slices of 2-week-old mice, they observed a total 
A
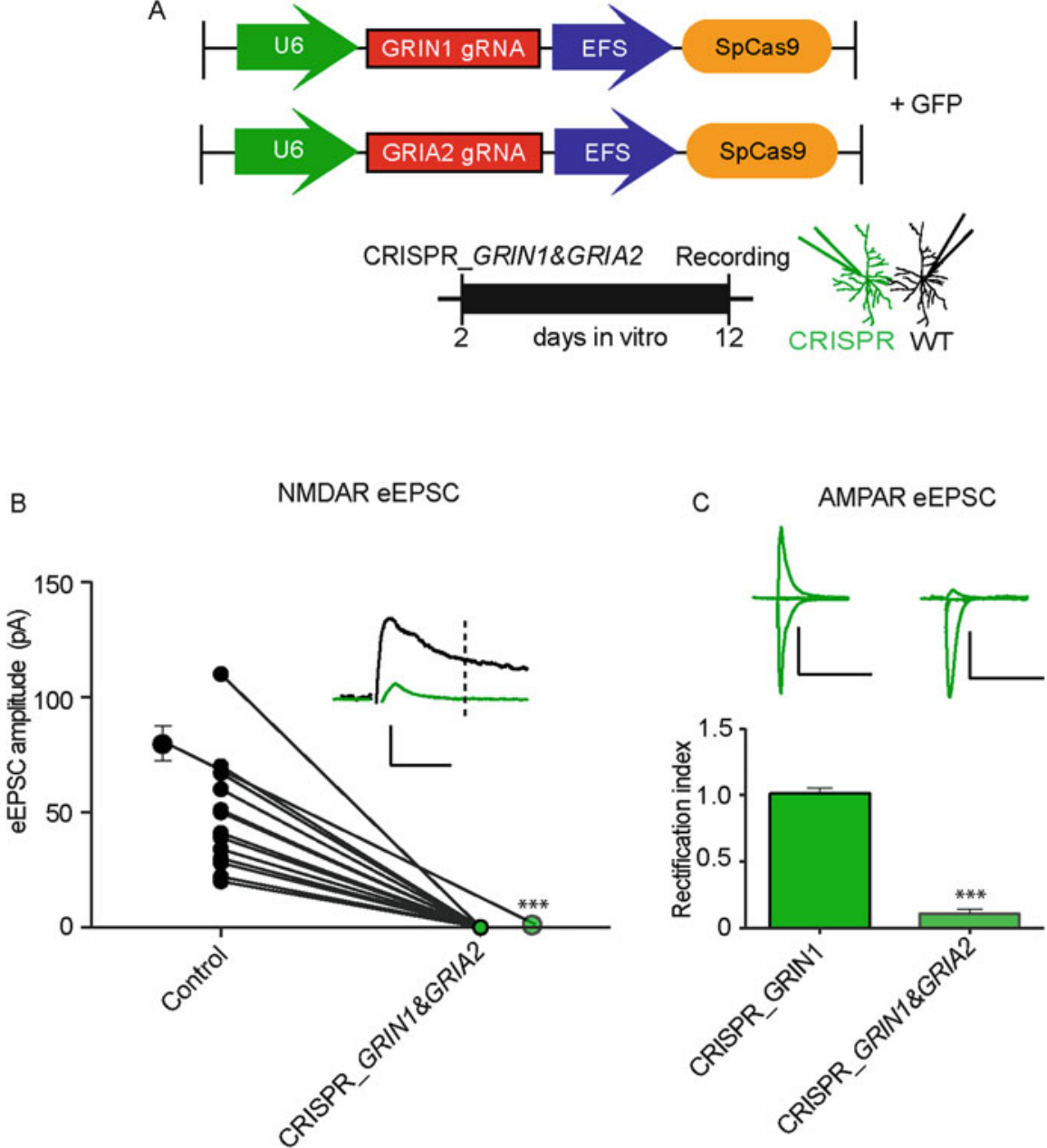

Fig. 3 (a) Scheme of the CRISPR plasmids modified to target specifically GRINI and GRIA2 and the time course of the transfection period before recording. (b) Sample trace and paired average NMDA-evoked EPSCs of single pairs from control and transfected cells. NMDA currents are completely eliminated after 10 days transfection. (c) AMPAR-evoked EPSCs summary of CRISPR_GRIN1 and double CRISPR_GRIN1\&GRIA2. Bar graph indicates the rectification index mean values for the two conditions. The double CRISPR conditions present a fully rectified phenotype typical of GluA1 monomeric receptors

elimination of NMDA currents with one guide RNA whereas the other guide RNA tested had no effect at all. This finding underlines the importance of guide RNA design and the necessity to validate these guide RNAs. In many ways, these considerations are not specific to CRISPR/Cas9 and are also true for Cre-Lox and RNAi approaches. 

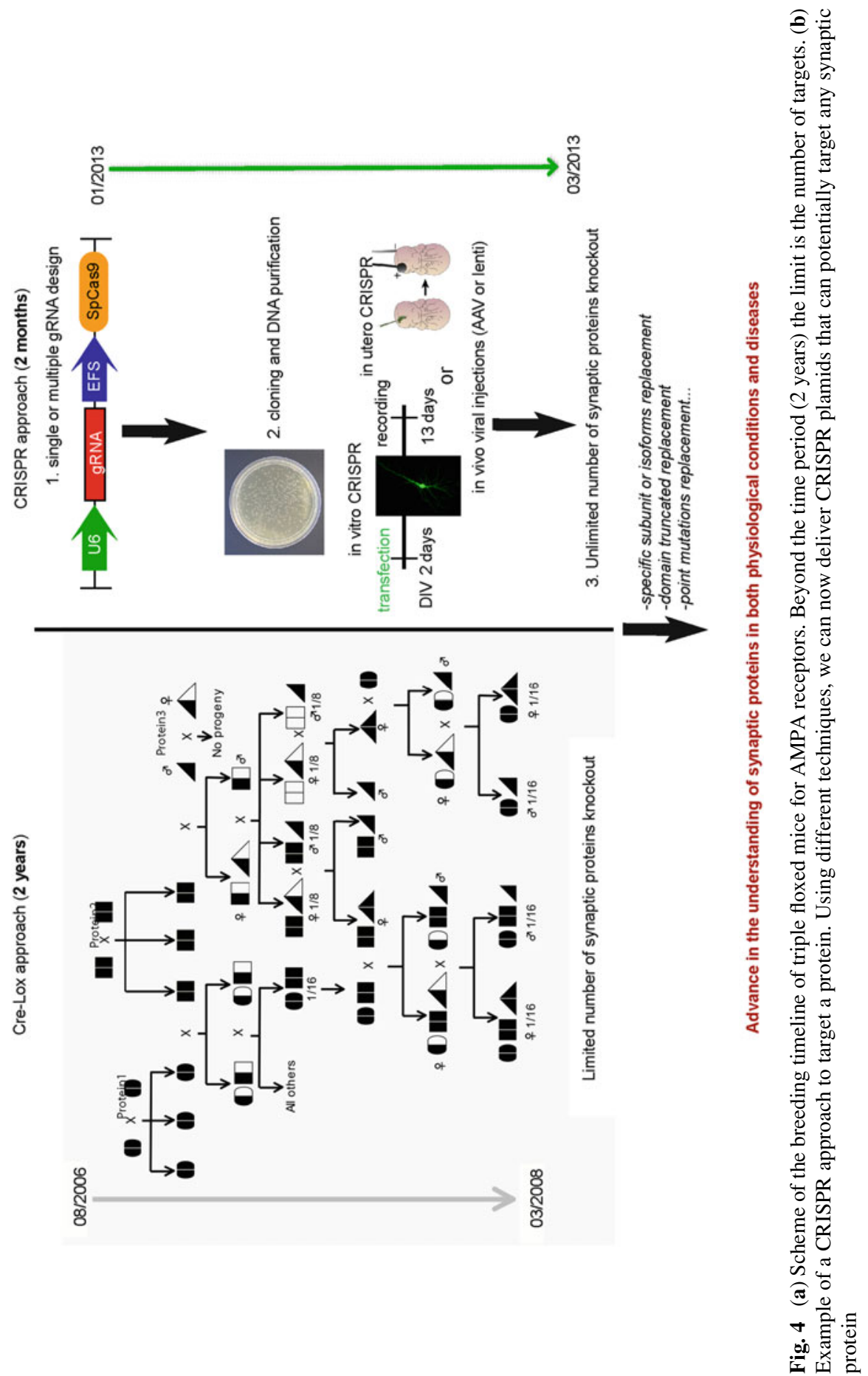
The generation of a Cre-dependent Cas9 knock-in mouse might also become a very useful tool for neuroscientists (Platt et al. 2014). By injecting AAV driving the expression of Cre and of a guide RNA targeting NeuN in the brain of the Cas 9 mouse, the authors observed the formation of on-target indels in the infected region accompanied by an $80 \%$ reduction in NeuN protein levels. By enabling the inactivation of genes either in vivo or in isolated primary cells, this mouse model will surely serve as a versatile tool and could potentially be used as a platform for genome-wide screens.

Finally, the combination of Cas9 with Sun-TAG technology enables the user to activate the expression of specific genes (Tanenbaum et al. 2014). The system is based on the recruitment of multiple copies of gene regulatory effector domains to a nuclease-deficient CRISPR/Cas9 protein targeted to specific sequences in the genome. CRISPR can thus be used not only to delete synaptic proteins but also to turn on their endogenous expression.

Most recent works on CRISPR/Cas9 systems evidence the importance of an optimized system. In particular modifications are due to the necessity of developing a possible human delivery system containing Cas9. Indeed, the switch to SaCas9 (from Staphylococcus aureus), which is much smaller, and the introduction of specific mutations to increase the specificity of Cas9 endonuclease cut, are examples of this race to new drug development (Ran et al. 2015). Furthermore, the introduction of specific mutations in the SpCas9 sequence has significantly enhanced the specificity of this enzyme. Thus, this improvement has reduced to a minimum the possibility of off-target effects, extending the applications of SpCas9 for genome editing (Slaymaker et al. 2016).

The use of CRISPR in neuroscience should be considered simply as a new tool, in particular for the time and cost reduction in the genetic manipulation of synaptic genes. In labs all around the world the introduction of CRISPR may not add anything really new regarding the final result but it can importantly simplify the work (Fig. 4).

Acknowledgments We thank R. H. Edwards, B. E. Herring, and F. Fieni for discussions and comments on the manuscript.

\section{References}

Adesnik H, Li G, During MJ, Pleasure SJ, Nicoll RA (2008) NMDA receptors inhibit synapse unsilencing during brain development. Proc Natl Acad Sci USA 105:5597-5602

Alvarez VA, Ridenour DA, Sabatini BL (2006) Retraction of synapses and dendritic spines induced by off-target effects of RNA interference. J Neurosci 26:7820-7825

Barnes DE (2001) Non-homologous end joining as a mechanism of DNA repair. Curr Biol 11: R455-R457

Bolotin A, Quinquis B, Sorokin A, Ehrlich SD (2005) Clustered regularly interspaced short palindrome repeats (CRISPRs) have spacers of extrachromosomal origin. Microbiology $151: 2551-2561$ 
Gray JA, Shi Y, Usui H, During MJ, Sakimura K, Nicoll RA (2011) Distinct modes of AMPA receptor suppression at developing synapses by GluN2A and GluN2B: single-cell NMDA receptor subunit deletion in vivo. Neuron 71:1085-1101

Han Y, Kaeser PS, Südhof TC, Schneggenburger R (2011) RIM determines Ca2+ channel density and vesicle docking at the presynaptic active zone. Neuron 69:304-316

Hayashi Y, Shi SH, Esteban JA, Piccini A, Poncer JC, Malinow R (2000) Driving AMPA receptors into synapses by LTP and CaMKII: requirement for GluR1 and PDZ domain interaction. Science 287:2262-2267

Herring BE, Shi Y, Suh YH, Zheng CY, Blankenship SM, Roche KW, Nicoll RA (2013) Cornichon proteins determine the subunit composition of synaptic AMPA receptors. Neuron 77:1083-1096

Hun S, Sharma M, Südhof TC, Shen J (2014) Synaptic function of nicastrin in hippocampal neurons. Proc Natl Acad Sci USA 111(24):8973-8978

Incontro S, Asensio CS, Edwards RH, Nicoll RA (2014) Efficient, complete deletion of synaptic proteins using CRISPR. Neuron 83:1051-1057

Ishino Y, Shinagawa H, Makino K, Amemura M, Nakata A (1987) Nucleotide sequence of the iap gene, responsible for alkaline phosphatase isozyme conversion in Escherichia coli, and identification of the gene product. J Bacteriol 169:5429-5543

Jansen R, Embden JD, Gaastra W, Schouls LM (2002) Identification of genes that are associated with DNA repeats in prokaryotes. Mol Microbiol 43:1565-1575

Jiang W, Bikard D, Cox D, Zhang F, Marraffini LA (2013) RNA-guided editing of bacterial genomes using CRISPR-Cas systems. Nat Biotechnol 31:233-239

Jinek M, Chylinski K, Fonfara I, Hauer M, Doudna JA, Charpentier E (2012) A programmable dual-RNA-guided DNA endonuclease in adaptive bacterial immunity. Science 337:816-821

Lieber MR (2010) The mechanism of double-strand DNA break repair by the nonhomologous DNA end-joining pathway. Annu Rev Biochem 79:181-211

Lu W, Shi Y, Jackson AC, Bjorgan K, During MJ, Sprengel R, Seeburg PH, Nicoll RA (2009) Subunit composition of synaptic AMPA receptors revealed by a single-cell genetic approach. Neuron 62:254-268

Makarova KS, Grishin NV, Shabalina SA, Wolf YI, Koonin EV (2006) A putative RNAinterference-based immune system in prokaryotes: computational analysis of the predicted enzymatic machinery, functional analogies with eukaryotic RNAi, and hypothetical mechanisms of action. Biol Direct 16:1-7

Maximov A, Pang ZP, Tervo DG, Südhof TC (2007) Monitoring synaptic transmission in primary neuronal cultures using local extracellular stimulation. J Neurosci Methods 161:75-87

Nakata A, Amemura M, Makino K (1989) Unusual nucleotide arrangement with repeated sequences in the Escherichia coli K-12 chromosome. J Bacteriol 171:3553-3556

Platt RJ, Chen S, Zhou Y, Yim MJ, Swiech L, Kempton HR, Dahlman JE, Parnas O, Eisenhaure TM, Jovanovic M, Graham DB, Jhunjhunwala S, Heidenreich M, Xavier RJ, Langer R, Anderson DG, Hacohen N, Regev A, Feng G, Sharp PA, Zhang F (2014) CRISPR-Cas9 knockin mice for genomic editing and cancer modeling. Cell 159:440-455

Pluck A (1996) Conditional mutagenesis in mice: the Cre/loxP recombination system. Int J Exp Pathol 77:269-278

Pourcel C, Salvignol G, Vergnaud G (2005) CRISPR elements in Yersinia pestis acquire new repeats by preferential uptake of bacteriophage DNA, and provide additional tools for evolutionary studies. Microbiology 151:653-663

Ran FA, Cong L, Yan WX, Scott DA, Gootenberg JS, Kriz AJ, Zetsche B, Shalem O, Wu X, Makarova KS, Koonin EV, Sharp PA, Zhang F (2015) In vivo genome editing using Staphylococcus aureus Cas9. Nature 520:186-191

Sauer B, Henderson N (1988) Site-specific DNA recombination in mammalian cells by the Cre recombinase of bacteriophage P1. Proc Natl Acad Sci USA 85:5166-5170 
Schnell E, Sizemore M, Karimzadegan S, Chen L, Bredt DS, Nicoll RA (2002) Direct interactions between PSD-95 and stargazin control synaptic AMPA receptor number. Proc Natl Acad Sci USA 99:13902-13907

Slaymaker IM, Gao L, Zetsche B, Scott DA, Yan WX, Zhang F (2016) Rationally engineered Cas9 nucleases with improved specificity. Science 351:84-88

Sternberg SH, Redding S, Jinek M, Greene EC, Doudna JA (2014) DNA interrogation by the CRISPR RNA-guided endonuclease Cas9. Nature 507:62-67

Straub C, Granger AJ, Saulnier JL, Sabatini BL (2014) CRISPR/Cas9-mediated gene knock-down in post-mitotic neurons. PLoS One 9(8):e105584. doi:10.1371/journal.pone.0105584

Tanenbaum ME, Gilbert LA, Qi LS, Weissman JS, Vale RD (2014) A protein-tagging system for signal amplification in gene expression and fluorescence imaging. Cell 159:635-646

Tsien JZ, Chen DF, Gerber D, Tom C, Mercer EH, Anderson DJ, Mayford M, Kandel ER, Tonegawa S (1996) Subregion- and cell type-restricted gene knockout in mouse brain. Cell $87: 1317-1326$

Wu X, Scott DA, Kriz AJ, Chiu AC, Hsu PD, Dadon DB, Cheng AW, Trevino AE, Konermann S, Chen S, Jaenisch R, Zhang F, Sharp PA (2014) Genome-wide binding of the CRISPR endonuclease Cas9 in mammalian cells. Nat Biotechnol 32:670-676

Open Access This chapter is licensed under the terms of the Creative Commons Attribution 4.0 International License (http://creativecommons.org/licenses/by/4.0/), which permits use, sharing, adaptation, distribution and reproduction in any medium or format, as long as you give appropriate credit to the original author(s) and the source, provide a link to the Creative Commons license and indicate if changes were made.

The images or other third party material in this chapter are included in the chapter's Creative Commons license, unless indicated otherwise in a credit line to the material. If material is not included in the chapter's Creative Commons license and your intended use is not permitted by statutory regulation or exceeds the permitted use, you will need to obtain permission directly from the copyright holder.

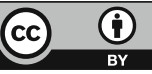

